Gynäkol Rundsch 1990;30:205

\title{
Grusswort des Verlegers
}

w12 Jahre Herausgebertätigkeit für die Gynäkologische Rundschau! Dafíir, lieber Herr Professor Gitsch, danke ich Ihnen im Namen der Her-ausgeber und als Verleger der Zeitschrift recht herzlich. In den Jahren unserer freundschaftlichen Zusammenarbeit hat sich die Zeitschrift zu einem im deutschen Sprachraum anerkannten Organ entwickelt. Ihre Arbeit in der Schriftleitung der Zeitschrift hat deren Inhalt wesentlich geprägt. In ausgezeichneter Weise haben Sie die Bedürfnisse der Leser, der Autoren und des Verlags aufeinander abgestimmt. Die Herausgabe des Berichts der Jahrestagung der «Österreichischen Gesellschaft für Gynäko-logie und Geburtshilfe», deren offizielles Organ die Gynäkologische Rundschau ist, hat Ihnen besonders am Herzen gelegen. Dieser Leistungsaus-weis der Gynäkologie in Forschung, Klinik und Praxis stellt einen bedeut-samen Teil der Zeitschrift dar. Die bei den Lesern so beliebten Kurzfassungen von Zeitschnftenartikeln haben Sie mit Ihren Mitarbeitern immer mit Sorgfalt ausgesucht und damit dem Bedürfnis Rechnung getragen, wis-senschaftliche Arbeiten über unsere Sprachgrenze hinaus zu vermitteln. Nicht zuletzt auch die Sektion «Fortbildung» wurde von Ihnen mit grosser Kompetenz betreut und hat das Erscheinungsbild der Zeitschrift nachhal-tig geprägt.

Auch wenn Sie sich nun aus der aktiven Redaktionsarbeit der Zeitschrift zurückziehen, so werden Sie bestimmt weiterhin an der Entwick-lung nicht nur auf dem Gebiet der Gynäkologie, sondern auch der Zeitschrift regen Anteil nehmen. Ihre Nachfolger werden Sie gerne auch in Zukunft konsultieren und ich bin überzeugt, dass die Zeitschrift in Ihrem Sinne weitergeführt wird. Für die Zukunft wünsche ich Ihnen im Namen der Schriftleitung und Redaktion sowie des Verlags das Allerbeste.

Thomas Karger 